\title{
Commentary: We came to an overwhelming and uniform opinion that these reports do not show colitis
}

There are no grounds to believe that any new inflammatory bowel disease was discovered by Wakefield et al

\author{
Ingvar Bjarnason professor of digestive diseases, consultant physician and gastroenterologist \\ King's College Hospital, London SE5 9RS, UK
}

The $B M J$ asked me to review 61 histology grading sheets completed by Amar Dhillon, ${ }^{1}$ relating to intestinal biopsies from 11 of 12 children reported by Wakefield and colleagues. ${ }^{2}$ I consulted several clinical and histopathology colleagues.

We came to an overwhelming and uniform opinion that these reports do not show colitis. Findings for all but one child represent changes within the normal appearance of bowel biopsies, and the changes reported for this child do not amount to colitis. The grading sheets make no provision for quantitative analysis, and the sheets themselves fall far below the standards expected for a research study.

We saw no evidence of enterocolitis, either histologically or in assumed association with reported endoscopic findings of ileal lymphoid nodular hyperplasia, which is a normal feature of young children that sometimes persists into adulthood. In my opinion, and that of those to whom I spoke, there is no justification whatsoever for calling this an enterocolitis.

The hospital pathology service found the histopathology to be normal, ${ }^{3}$ and, except in the case of the child mentioned above, the grading sheets also note normal findings. The fact that these scores were interpreted as abnormal raises, in my opinion, questions for the authors of Wakefield et al to answer, and particularly for the consultant histopathologists.
From the histological and endoscopic reports, there are no grounds to believe that any new inflammatory bowel disease may have been discovered, or any possible "unique disease process" observed, as reported by Wakefield et al. Nothing can be said about the aetiology of any minor irritations noted, and nothing can be inferred regarding treatment..

Competing interests: All authors have completed the ICMJE uniform disclosure form at www.icmje.org/coi_disclosure.pdf (available on request from the corresponding author) and declare: no support from any organisation for the submitted work; no financial relationships with any organisations that might have an interest in the submitted work in the previous three years; no other relationships or activities that could appear to have influenced the submitted work.

Provenance and peer review: Commissioned, not externally peer reviewed.

1 Godlee F. Institutional research misconduct. BMJ 2011:343;7284.

2 Wakefield AJ, Murch SH, Anthony A, Linnell J, Casson DM, Malik M, et al. lleal lymphoid nodular hyperplasia, non-specific colitis and pervasive developmental disorder in children. Lancet 1998;351:637-41 [retracted].

3 Deer B. Wakefield's "autistic enterocolitis" under the microscope. BMJ 2010;340:c1127. 